\title{
Measuring system for liquid level determination based on linear electro-optical effect of liquid crystal
}

\author{
Olga Denisova* \\ Ufa State Petroleum Technological University, Department of Physics, 450062 Kosmonavtov Str. 1, \\ Russia
}

\begin{abstract}
This article describes an updated system for measuring and controlling the level of liquid media. Well-known capacitance method for determining the liquid level is modernised. The new scheme proposes the use of electro-optical cell with a nematic liquid crystal. Homeotropically oriented liquid crystal is sandwiched between two plates, one of which is glass, and the other - crystal - cadmium sulfide $C d S$ photoconductor. liquid crystal cell serves as an indicator. Its light transmittance depends on the applied voltage. Cell is designed so that the dependence of the phase delay of the voltage is linear. The article describes a mathematical model showing linear dependence, confirmed experimentally. Application of linear electrooptic effect observed in liquid crystals, allows to improve the accuracy and speed of measurement of liquid media, as the liquid crystal is an anisotropic medium more sensitive than solid crystals. The relaxation time of the orientation effects in liquid crystals is $\sim 10^{-6} \mathrm{~s}$. From the point of view of practical significance, this method will be of interest for application in the fuel and energy complex, in particular, oil and gas industry for the commercial accounting of petroleum products.
\end{abstract}

\section{Introduction}

Despite the fact that liquid crystals were discovered. At present, ever higher requirements are imposed on the accuracy of measurements. This is due to the fact that the issue of measuring the liquid level is actual in many branches of the national economy, especially in the oil and gas industry with the commercial accounting of refined products.

There is a variety of instruments and systems for measuring the liquid level, which are based on various physical phenomena at the moment [1]. Increasing demands are placed on the reliability and speed of these systems, which requires new scientific and technical solutions. The authors [2-9] have experimentally and theoretically studied various effects observed in liquid crystals, which can form the basis for the development of sensors used for various purposes. It is well known that liquid crystals (LC) are very sensitive to external influences, which will significantly improve the efficiency of measuring systems. The principle of measuring systems operation is based on a huge variety of orientation effects

* Corresponding author: denisovaolga@bk.ru 
that are observed when electric, magnetic, thermal, acoustic fields are affected by liquid crystals, as well as under the mechanical action of shear, stretching, torsion. In addition to the information display systems, liquid crystals are already used to indicate various parameters in engineering and medicine (for the detection of harmful chemical compounds, non-destructive testing methods, pressure sensors for underwater sounding, such as electrooptical switches, for vibration control).

\section{Description of the method}

Many technological processes require the control of the level of various liquids. To solve this problem, enterprises specializing in the manufacture of instrumentation, offer level controllers, as well as float sensors, which can be used both for working with controllers and for controlling actuators directly. Instruments for measuring the level of liquids and solids can work on a mechanical, hydrostatic, electrical, magnetic or optical principle or use level gauges with echolocation and radar. Level controllers and sensors are universal precision devices designed for a wide range of applications where an ultra-precise flow measurement is required. They can be used to solve the following tasks: maintaining the liquid level within the specified limits; control of pumps filling storage or pressure tanks, chemical reactors; Control of pumps supplying water from wells and pumping it out of different capacities; protection from dry running of pumps, electric heaters and emergency shutdown of appliances, the operation of which is inadmissible without water (heating electric boilers, water heaters); prevention of overflow and pollution; monitoring the level of fluid in vehicles, for example, controlling the volume of fuel in heavy equipment (trucks, excavators, diesel locomotives).

The article offers a highly effective method for measuring the level of liquid media. Based on the already known capacitive method for determining the level of liquid or bulk substances. But the essence of the method of indication is the use of a linear electro-optic effect in nematic liquid crystals [2-9]. An electro-optical effect is the change in the refractive index of a substance under the action of an electric field. They are linear and quadratic. In solid crystals (for example, glass) distinguish the Pockels effect - a linear electro-optic effect. It is observed when the change in the refractive index of a substance is directly proportional to the first degree of intensity of the applied external electric field $\Delta n \sim E$. The Kerr effect or the quadratic electro-optic effect occurs when the change in the refractive index is directly proportional to the square of the intensity of the acting electric field $\Delta n \sim E^{2}$.

An electric birefringence (Kerr effect) in the melt of a comb-like polymer with an acrylic backbone and mesogenic cyanobiphenyl side groups at a temperature above the smectic isotropic phase transition was investigated [10]. It was found that the Kerr constant varies abruptly as a function of temperature, which is due to a change in the short-range orientation order.

The use of the liquid crystal cell in the proposed system gave an increase in the speed and accuracy of measurements, and increased its functionality, which became possible due to an evident orientation effect in liquid crystals, which relaxation time is $10^{-6} \mathrm{~s} \mathrm{[2-9].}$

A coaxial capacitor the electrodes of which are connected to the liquid crystal cell is used in this structural scheme (Figure 1). It is made in the form of a hollow cylindrical electrode 1 and a central electrode - rod 2, connected to the electrodes of the electro-optical liquid crystal cell. The cell was illuminated by a laser 3 whose beam first passed through the polarizer 4 . Then, the light that passed through the cell 5 and the analyzer with the photodetector 6 is processed by the microcontroller 7 , which includes an amplifier, an analog-to-digital converter, and a microprocessor. The data from the microcontroller goes to the operator's automated workstation and displayed on screen monitors 8 in any convenient form. The 
principle of operation of the electro-optical system on nematic liquid crystals is as follows: a change in the level of the dielectric liquid between the coaxial measuring capacitor between electrodes 1 and 2 causes a change in the capacitance of this capacitor in proportion to the change in the liquid level. This, in turn, leads to a change in the reactance of the capacitor and leads to a change in the voltage drop on it.

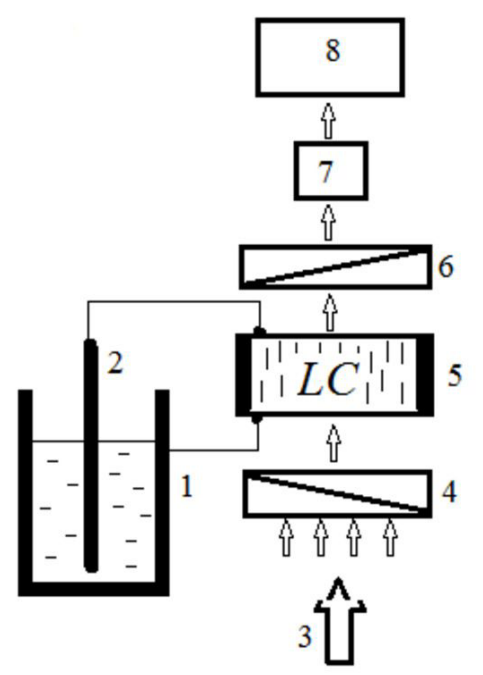

Fig. 1. Structural diagram of a measuring system based on a linear electro-optical effect in nematicliquid crystal: 1 - hollow cylindrical electrode, 2 - central electrode - rod, 3 - laser, 4 polarizer, 5 - liquid crystal cell, 6 - analyzer with photodetector, 7 - microcontroller, which includes amplifier, analog-digital converter and microprocessor, 8 - monitor screen.

The LC-cell (Figure 2) is a system of conductive glass, the crystal-photoconductor $C d S$. The $\mathrm{SnO}_{2}$ tin dioxide electrodes were attached to the plates. The use of $C d S$ is due to the fact that it is a photoconductor, and its conductivity can be varied with the help of light of different intensity. A mixture of a nematic liquid crystals of $n$ - $n$-butyl- $n$-heptanoyloxybenzene and $n$ - type - methoxybenzylidene - $n$ - butylaniline was placed between the plates.

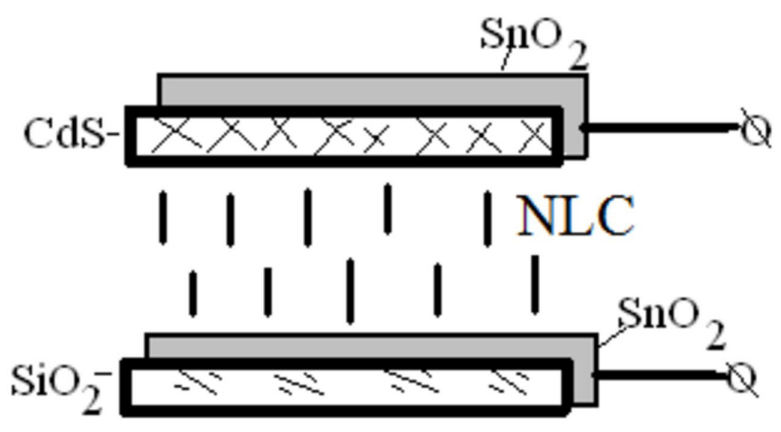

Fig. 2. Experimental electro-optical liquid crystal cell.

Liquid crystal molecules had a homeotropic orientation. This orientation was obtained as a result of ion bombardment with argon of $\mathrm{SnO}_{2}$ and $\mathrm{CdS}$ surfaces. The phase delay of light passing through the liquid crystal cell is linearly dependent on the voltage applied to the cell plates [11-15]. 


\section{Result and discussion}

A mathematical model of the linear electro-optic effect observed in nematic liquid crystals. Since the nematic has a permittivity $\varepsilon_{a} \approx 0$, it can be considered semi-infinite, provided that at the thickness of the liquid crystal $z \rightarrow \infty$ the angle of inclination of the liquid crystal molecules is $\theta \rightarrow 0$.

Taking into account the long-range order parameter:

$$
S_{1}^{*}(z)=S_{10} \frac{S h(-\lambda z)}{S h(\lambda h / 2)}+S_{1 \infty},
$$

where $S_{10}$ - order parameter near the surface in the absence of the field, $h$ - the thickness of the liquid crystal,

$$
\lambda=\left(\frac{C_{3}}{\alpha T^{\prime}-C_{1}}\right)^{-1 / 2},
$$

that is the distribution of the dipole moment $p$ decreases from the electrode into the interior of the liquid crystal, as

$$
|p|=p_{o} S_{l}=p_{o} S_{10} e^{b z}, \quad b=-1 / \delta,
$$

$\delta \sim \lambda^{-1}$ - effective depth of existence of surface polarization,

$$
C_{1}=\frac{a}{2} \quad \text { and } \quad C_{3}=-\lambda e^{-k \beta}-\frac{a}{2}\left(1+e^{-2 k \beta}\right)
$$

- constants, where $a=\frac{p_{o} S_{10} E}{k}, k$ - elastic coefficient, $E$ - electric field strength.

The equation of a nematic liquid crystal state will have the form:

$$
k \theta_{x x}+E p_{o} S_{10} e^{-\lambda z} \theta=0
$$

We introduce the notation, $y=\eta(\xi), \xi=e^{-\lambda z}$, we obtain the equation:

$$
\lambda^{2} \xi \frac{d^{2} \eta}{d \xi^{2}}-\lambda \frac{d \eta}{d \xi}+a \eta=0
$$

Solution of equation

$$
\begin{gathered}
\eta[\theta]=\eta_{O}\left[\theta_{O}\right]\left(d^{\prime}-1\right)\left(d^{\prime}-3\right) \ldots\left(d^{\prime}-2 n+1\right) e^{ \pm^{V z}} \\
d^{\prime}=z \frac{d}{d z}, v=1 / c=-\lambda / a
\end{gathered}
$$

further, for $a<<1$, we obtain for the change in the refractive index

$$
\Delta n \sim \frac{p_{o} S_{10} E}{k}=\frac{p_{o} S_{10} U}{k h},
$$

That is, the change in the refractive index of the liquid crystal depends on the applied voltage of the electric field in direct proportion, which corresponds to figure $3 b$. 
Figure 3 shows the curves for the phase delay and the intensity of light passed through the liquid crystal cell (wavelength $550 \mathrm{~nm}$ ) as a function of the voltage. They describe with a high accuracy $(<10 \%)$ (Figure $3 \mathrm{~b})$ the linear variation of the birefringence of the cell (1). The value of the angle of inclination of liquid crystal molecules $\theta_{o}$ is determined, from the condition of weak adhesion on the surface, by the expression $\boldsymbol{h} \times \boldsymbol{g}$ [11-15]. According to the proposed mathematical model, in the presence of boundaries and anisotropic interaction of liquid crystal molecules with a solid substrate in a nematic liquid crystal, a polarized layer-a surface polarization with an effective thickness of $\delta \sim \lambda^{-1}$ is formed. When an electric field is applied to the nematic liquid crystal, a polarization is induced directly proportional to the order parameter $P \sim S_{l}$, which magnitude in the fields $E \approx 5 \cdot 10^{4} \mathrm{~V} / \mathrm{cm}$ is $P_{V} \approx 10^{1}-10^{2}$ units CGSE $/ \mathrm{cm}^{3}$, and the value of the long-range dipole order in this case is $2 \cdot 10^{2}-5 \cdot 10^{2}$.

a)

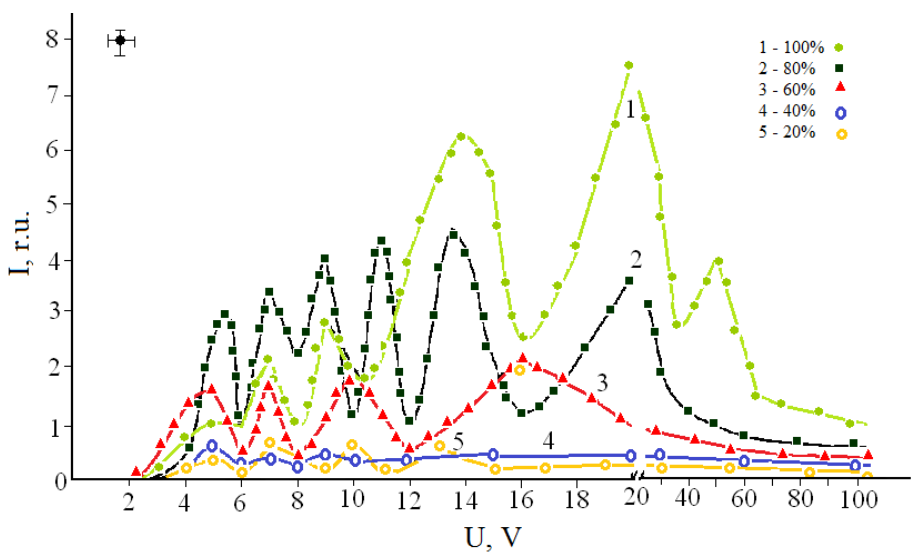

b)

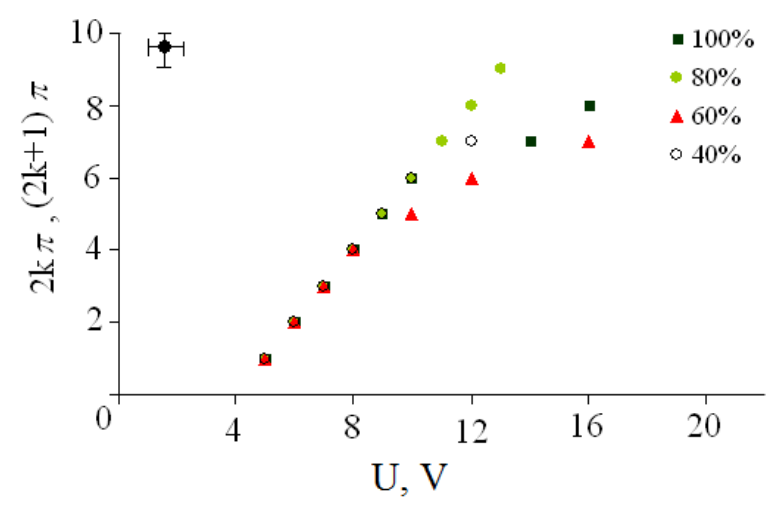

Fig. 3. Dependences a) of the optical signal on the voltage for various values of the substrate illumination; b) of the phase delay from the voltage, the value of the substrate illumination (in percent).

\section{Conclusion}

The main reason for measuring the level is the need to track the number of products in units of volume or mass. Industrial requirements for technological accounting are constantly toughened. Level measurement is one of the key components of the tank farm accounting system to ensure reliable and accurate management of raw materials and finished products. Over the past few years, stock accounting has acquired special significance not only for 
operational personnel, but for companies in general, including managers and persons responsible for material accounting and analysis of unproductive losses. This is the result of increased attention to safety issues and product costs.

In many cases, the calculation of the quantity of the product to be bought or sold (transferred to responsible storage) is based on the value of the product level, according to which either volume or mass is calculated. In case of commercial accounting, the requirements for the level gauge error are very high, since an error of the order of $3 \mathrm{~mm}$ can lead to a very significant error in calculating the volume. For the account, devices of an approved sample should be used, the accuracy of which, as a rule, exceeds $1 \mathrm{~mm}$.

Accurate level measurement improves efficiency. If a certain quantity of raw materials is needed in the tank farm and the tanks do not use their full capacity, the company will bear the cost of purchasing and maintaining additional storage tanks. Effective use of the volume prevents additional costs for the purchase of additional tanks.

Level measurement is also carried out for the purpose of ensuring safety. Filling open tanks in excess of the calculated capacity can create a safety hazard - overfilling. If corrosive, reactive, combustible or toxic materials are stored in tanks, overflowing or creating excessive pressure can cause catastrophic consequences. In tanks with products of this type, it is also important to monitor the level to ensure that there are no leaks. In addition, preventing overflows and detecting leaks is important to meet the requirements of environmental legislation.

In many technological processes, it is necessary to ensure a uniform supply of the product at the inlet and outlet. Ensuring a constant supply can be difficult due to variations in the flow velocity or pressure in the supply line. If the technological level in the storage tank is constantly maintained within a predetermined range, the feed rate at the inlet of the reservoir can increase and decrease without affecting the rate of flow from the reservoir to the process. The constant supply directly affects the quality of products in the pulp and paper industry, since this ensures the same thickness of all produced sheets of paper.

In summary, the work carried out showed the spread of Thus, the article suggests a modernized capacitive system for monitoring and changing the level of liquid media. In this system, a liquid crystal cell is used as an indicator, and its application makes it possible to increase the efficiency of measurements, i.e. speed, sensitivity of the method, as well as its functionality. This method can be used in the fuel and energy complex.

\section{References}

1. G.G. Rannev, Methods and means of measurement (Academia, Moscow, 2008)

2. O.A. Denisova, A.N. Chuvyrov, Liq. Cryst. and their Appl., 2 (2011)

3. O.A. Denisova, The nonequilibrium structural changes of liquid crystals in electric fields and currents acoustic (UGAES, Ufa, 2012)

4. O.A. Denisova, Electronic scientific journal "Oil and gas business", 5 (2011)

5. O.A. Denisova, O.A. Skaldin. Letters on materials, 6 (3) (2016)

6. O.A. Baimakova, O.A. Skaldin, A.N. Chuvyrov, Mol. Cryst. Liq. Cryst., 265 (1995)

7. O.A. Denisova, A.N. Chuvyrov, Liq. Cryst. and their Appl., 1 (2012)

8. O.A. Denisova, A.N. Chuvyrov, Liq. Cryst. and their Appl., 2 (2011)

9. O.A. Denisova, A.N. Chuvyrov, Liq. Cryst. and their Appl., 3 (2011)

10. A.M. Alekseev, A.I. Buzin, Soros Educational Journal, 3 (1998)

11. O.A. Denisova, A.N. Chuvyrov, Bashkirsky Chemical Journal, 18 (3) (2011)

12. O.A. Denisova, Electrical and information complex and systems, 9(4) (2013)

13. V.V. Leonov, O.A. Denisova, Electrotechnical and Information Systems and Systems, 2 (2015) 
14. O.A. Denisova, A.N. Chuvyrov, Liq. Cryst. and their Appl., 2 (44) (2013)

15. O.A. Denisova, R.L. Abramishvili, Electrical and information complex and systems, 13(1) (2017) 